\title{
The classification of tight contact structures on the 3-torus \\ KANDA YUTAKA
}

In this paper, we obtain the classification of orientable tight contact structures on the 3-torus.

\section{Introduction.}

A contact structure $\zeta$ on a 3 -manifold $M$ is called tight if the characteristic foliation of any embedded disc $D$ has no limit cycle, and $\zeta$ is called overtwisted if otherwise.

The classification of over twisted contact structures coincides with the classification of homotopy classes of 2-plane fields. See [E1].

In contrast, the classification of tight contact structures is much more complicated. For example, it was known that $T^{3}$ has at least two positive (resp. negative ) tight contact structures which are not isotopic, but belong to the same homotopy class. See [G2].

In this paper, we obtain the following results:

(1) Theorem 0.1. For any positive integer $n$, let $\xi_{n}$ denote the tight contact structure on the 3-torus $T^{3}$ defined by the 1-form $\cos 2 \pi n z d x+$ $\sin 2 \pi n z d y$. There is no contact diffeomorphism between $\left(T^{3}, \xi_{n}\right)$ and $\left(T^{3}, \xi_{m}\right)$ if $n \neq m$.

(2) Theorem 0.2. Any contact diffeomorphism of $\left(T^{3}, \xi_{n}\right)$ is isotopic to an automorphism which preserves the 1-form $d z$. Conversely, any automorphism of $T^{3}$ which preserves $d z$ is isotopic to a contact diffeomorphism with respect to $\xi_{n}$.

(3) Theorem 0.3. For any tight contact structure $\left(T^{3}, \zeta\right)$, there is a positive integer $n$ such that $\left(T^{3}, \zeta\right)$ is contact diffeomorphic to $\left(T^{3}, \xi_{n}\right)$.

The main tools used in this paper is the theory of characteristic foliations and convex surfaces developed by Giroux in [G1]. Thanks to this theory, 
we can introduce the cut and paste method to the study of cortact structures. Fortunately, the tight contact structure on the closed 3-ball $B^{3}$ is completely determined by the characteristic foliation induced on $\partial B^{3}$. See [E2]. Therefore, we can reduce the situation to the case of $B^{3}$ by finding "good" convex surfaces in the tight contact 3-manifold $M$ and splitting $M$ along them.

The key in this operation is to pay great attention to simple closed Legendrian curves. If a simple Legendrian curve $s$ and a contact structure $\zeta$ are given in a small tubular neighborhood of $s$, there often exists a strict restriction to extend $\zeta$ to the whole $M$ as a tight contact structure.

This paper is organized as follows:

Section 1,2 and 3 are devoted to the summary of several known results.

Section 4,5 and 6 are devoted to prepare several tools necessary in Section 8.

In Section 7, we introduce the invariant of $\left(T^{3}, \xi_{n}\right)$. Theorem 0.1 and 0.2 are easily proven by using this invariant.

In Section 8, we prove Theorem 0.3.

Note. The author would like to thank E.Giroux who kindly sent him his preprint [G2]. E.Giroux informed us that he also obtained the classification of tight contact structures on the 3-torus and gave lectures on it in the international symposium in honor of Cerf in May,1994 and in a colloquium at Cambridge.

Remark. In this paper, for a set $A$, the symbol \#A means the number of components of $A$.

\section{Contact Structures.}

A contact structure $\zeta$ on a 3-manifold $M$ is a completely non-integrable 2-plane field.

If $\alpha$ denote a locally defined 1 -form which defines $\zeta$ (i.e, $\left.\alpha\right|_{\zeta}=0$ and $\alpha$ is non-singular.), the 3-form $\alpha \wedge d \alpha$ does not vanish anywhere. If one changes the sign of $\alpha, \alpha \wedge d \alpha$ does not alter its sign. Therefore, there is the canonical orientation of $M$ determined by $\zeta$. We call $\zeta$ positive if this orientation coincides with the given orientation of $M$, and negative if otherwise.

We say that $\zeta$ is orientable if $\zeta$ is orientable as a 2-plane field. 
Contact structures do not have any local invariant. i.e, for any point $x \in M$, there is a neighborhood $U$ of $x$ and a injective map $\Phi: U \rightarrow \mathbb{R}^{3}$ such that $\Phi(x)=0$ and that $\Phi_{*}(\zeta)$ coincides with the standard contact structure of $\mathbb{R}^{3}$ defined by the 1 -form $d z+x d y$.

\section{Tight Contact Structures.}

Definition 2.1. A contact 3-manifold $(M, \zeta)$ is tight if the characteristic foliation of any embedded disc in $M$ has no limit cycle.

Example 2.2. $\left(\mathbb{R}^{3}, d z+x d y=0\right)$ is a tight contact structure and called the standard contact structure of $\mathbb{R}^{3}$.

Example 2.3. Let us identify $S^{3}$ with $S U(2)$. Take three left invariant vector fields $X, Y, Z$ on $S U(2)$ such that $[X, Y]=Z,[Y, Z]=X,[Z, X]=Y$. The 2-plane field $\xi_{0}$ spanned by $X$ and $Y$ is a tight contact structure, which is called the standard contact structure of $S^{3}$.

Example 2.4. Let us identify the 3 -torus $T^{3}$ with $\mathbb{R}^{3} / \mathbb{Z}^{3}$. For any positive integer $\mathrm{n}$, the contact structure $\xi_{n}$ on $T^{3}$ defined by the 1-form $\cos 2 \pi n z d x+$ $\sin 2 \pi n z d y$ is a tight contact structure. See [G2].

Theorem 2.5 [E2]. Two positive tight contact structures on the 3-ball $B^{3}$ which induce the same characteristic foliation $\mathfrak{F}$ on $\partial B^{3}$ are isotopic through an isotopy which preserves each leaf of $\mathfrak{F}$.

\section{Characteristic Foliations, Contact Vector Fields and Convex Surfaces.}

We summarize here several known results which we will need in the following sections.

Definition 3.1. Let $F$ be an orientable surface. A singular foliation is an equivalence class of flows on $F$ where the equivalence relation is as follows:

"Two flows $X$ and $Y$ are equivalent if and only if there is a positive function $f$ such that $X=f Y$."

Let $[Y]$ denote the equivalence class which $Y$ belongs to, and we say that $Y$ generates $[Y]$.

Definition 3.2. Let $F$ be an oriented surface embedded in an oriented contact 3-manifold $(M, \zeta)$. Let $Y$ be the flow on $F$ defined by the equation 
$i(Y) \omega=\left.\alpha\right|_{F}$, where $\alpha$ is a global 1-form which defines $\zeta$, and $\omega$ is a volume form on $F$.

The characteristic foliation $\mathfrak{F}$ on $F$ induced by $\zeta$ is the singular foliation generated by $Y$. We will denote the characteristic foliation induced on $F$ by $c h_{\zeta}(F)$.

The geometric meaning of characteristic foliations is shown in the following Proposition.

Proposition 3.3 [G1]. Suppose that $\operatorname{ch}_{\zeta}(F)$ is generated by a flow $Y$. Then $Y$ is tangent to $\zeta$ and the singularities $Y$ coincide with the tangencies of $T F_{x}$ and $\zeta_{x}$.

Intuitively speaking, the characteristic foliation on $F$ is the one obtained by integrating the line field $F \cap \zeta$.

Proposition 3.4 [G1]. Let $F$ be a closed orientable surface ana! denote by $F_{0}$ the submanifold $F \times\{0\}$ of $F \times \mathbb{R}$.

Let $\mathfrak{F}$ be a singular foliation on $F_{0}$. Then:

(1) $\mathfrak{F}$ is induced by a germ of contact structure along $F_{0}$ if and only if $\mathfrak{F}$ is generated by a flow $Y$ whose divergence does not vanish on the singular set of $Y$.

(2) Let $\zeta_{1}$ and $\zeta_{2}$ be germs of positive contact structures along $F_{0}$ such that both $\zeta_{1}$ and $\zeta_{2}$ induce the same characteristic foliation $\mathfrak{F}$ on $F_{0}$. Then there is a germ of diffeomorphism $\Psi$ such that $\Psi\left(\zeta_{1}\right)=\zeta_{2}$ and such that $\Psi$ is isotopic to the identity through an isotopy which, preserves each leaf of $\mathfrak{F}$.

Definition 3.5. A flow $X$ on $(M, \zeta)$ is contact if its 1-parameter group $\Phi_{X}^{t}$ preserves $\zeta$.

Proposition 3.6 $[\mathbf{A}],[\mathbf{W}]$. A contact flow on $(M, \zeta)$ is identijied with a section of the bundle $T M / \zeta$.

Precisely speaking, $X_{x}$ is determined by the 1-jet at $x$ of the corresponding section of $T M / \zeta$.

Definition 3.7. An orientable surface $F$ embedded in a contact 3-manifold $(M, \zeta)$ is convex if there is a contact flow $X$ transverse to $F$.

Theorem 3.8 [G1]. Let $F$ be a closed orientable surface enzbedded in $(M, \zeta)$. We can perturb $F$ by $a C^{\infty}$-small isotopy so that $F$ is convex. 
If $F$ is a convex surface in $(M, \zeta)$, there is a vertically invariant contact structure $\zeta^{\prime}$ on $F \times \mathbb{R}$ such that the map $\Psi: F \times \mathbb{R} \rightarrow M$ defined by $\Psi(x, t)=\Phi_{X}^{t}(x)$ is a contact embedding with respect to $\zeta^{\prime}$ and $\zeta$. Thus, we have only to study vertically invariant contact structures to know the properties of convex surfaces.

Notice that Proposition 3.4 implies that the convexity of $F$ is completely determined by the characteristic foliation on $F$.

Definition 3.9. Let $\mathfrak{F}$ be a singular foliation on a closed orientable surface $F$.

We say that an disjoint union of simple closed curves $\Gamma \subset F$ divides $\mathfrak{F}$ if $\Gamma$ satisfies the following properties:

(1) $\Gamma$ divides $F$ into two parts $F^{+}$and $F^{-}$such that $\Gamma=\partial F^{+}=-\partial F^{-}$. We call $F^{+}$(resp. $F^{-}$) the positive (resp. negative) part of $F$.

(2) $\Gamma \pitchfork \mathfrak{F}$.

(3) There are a flow $Y$ and a volume form $\omega$ on $F$ such that:

1. $Y$ represents $\mathfrak{F}$.

2. $L_{Y} \omega>0$ on $F^{+}$and $<0$ on $F^{-}$.

3. $\left.Y\right|_{F^{+}}$goes outward along $\partial \overline{F^{+}}$.

Remark. Any two dividing sets of $\mathfrak{F}$ can be connected by a 1-parameter family of dividing sets of $\mathfrak{F}$.

Theorem 3.10 [G1]. A closed orientable surface $F$ embedded in $(M, \zeta)$ is convex if and only if there exist a dividing set $\Gamma$ of $\operatorname{ch}_{\zeta}(F)$.

Precisely speaking, what the above theorem assarts is as follows:

Suppose that there is a contact flow $X$ transverse to $F$. Define the characteristic manifold $C(X)$ by $C(X):=\{x \in$ $M \mid X_{x}$ is tangent to $\zeta_{x}$ and $\left.X_{x} \neq 0\right\} . C(X)$ is a submanifold of codimension 1 of $M$, intersects $F$ transversely and oriented canonically by $\zeta, X$ and $F$. Then $c h r_{\zeta}(F)$ is divided by $\Gamma_{F}^{X}$.

Conversely, if a given set $\Gamma$ divides $c h_{\zeta}(F)$, then there is a contact flow $X$ transverse to $F$ such that $C(X) \cap F$ coincides with $\Gamma$ including their orientations. 
Notation. Let $F^{+, X}$ and $F^{-, X}$ denote the positive and the negative part of $F$ determined by $C(X) \cap F$, respectively. We omit $X$ from these symbols when we need not refer to the contact flow $X$.

Definition 3.11. Let $X$ be a contact flow on $(M, \zeta)$ and $F$ a convex surface transverse to $X$.

We say an isotopy $H_{t}$ of $F$ admits $X$ or is admissible with respect to $X$ if $H_{t}$ is transverse to $X$ for all $t$.

Theorem 3.12 [G1]. Let $f: F \rightarrow(M, \zeta)$ be an embedding of a closed surface such that $f(F)$ be convex. Let $\Gamma$ be a dividing set of the singular foliation $f^{-1}\left(\operatorname{ch}_{\zeta}(f(F))\right)$ denoted by $\mathfrak{F}_{0}$.

Suppose that another singular foliation $\mathfrak{F}_{1}$ be divided by $\Gamma$. Then there is an admissible isotopy $H_{t}$ such that:

(1) $H_{0}=f$

(2) $H_{1}^{-1}\left(\operatorname{ch}_{\zeta}\left(\operatorname{Im} H_{1}\right)\right)=\mathfrak{F}_{1}$,

(3) $H_{t}^{-1}\left(\left(I m H_{t}\right)^{0}\right)=\Gamma$ for all $t$,

(4) If there is an open set $U \subset F$ such that $\left.\mathfrak{F}_{0}\right|_{U}=\left.\mathfrak{F}_{1}\right|_{U}$, then we can choose $H_{t}$ so that $\left.H_{t}\right|_{U}=\left.f\right|_{U}$ for all $t$.

Notice that if $X$ is a contact flow transverse to $F$, the admissible isotopy $H_{t}$ with respect to $X$ satisfies that $\operatorname{Im} H_{t} \subset \operatorname{supp}(X)$ for all $t$.

Of course, $\left\{H_{t}\right\}$ themselves cannot be taken $C^{0}$-close to $f$ in many cases even if $\operatorname{supp}(X)$ is contained in a $C^{0}$-small neighborhood of $F$. But the following Proposition 3.13 gives us a way of eliminating singularities of characteristic foliations by a $C^{0}$-small isotopy.

We call a singular point $p$ of a characteristic foliation positive (resp. negative) if the orientations of $T F$ and $\zeta$ coincide (resp. are opposite) at $p$. The positivity of a singular point $p$ is equivalent to the condition that if a flow $Y$ generates the characteristic foliation, $\operatorname{div}_{\omega} Y$ is positive at $p$. Notice that any singular point of a characteristic foliation is either positive or negative.

Proposition 3.13 [G1],[E2]. Let $F$ be a closed orientable surface in $(M, \zeta)$ and $c_{\zeta}(F)$ generic. Let $l$ be a separatrix whose ends consist of one positive elliptic point e (i.e. its index $=1$ ) and one positive hyperbolic point $h$ (i.e. its index $=-1$ ). Let $U$ be sufficiently small neighborhood of $l$ in $F$. Then we can perturb $F$ with a $C^{0}$-small isotopy which fixes $F-U$ so that $c h_{\zeta}(U)$ is non-singular. 
Figure 4.1.
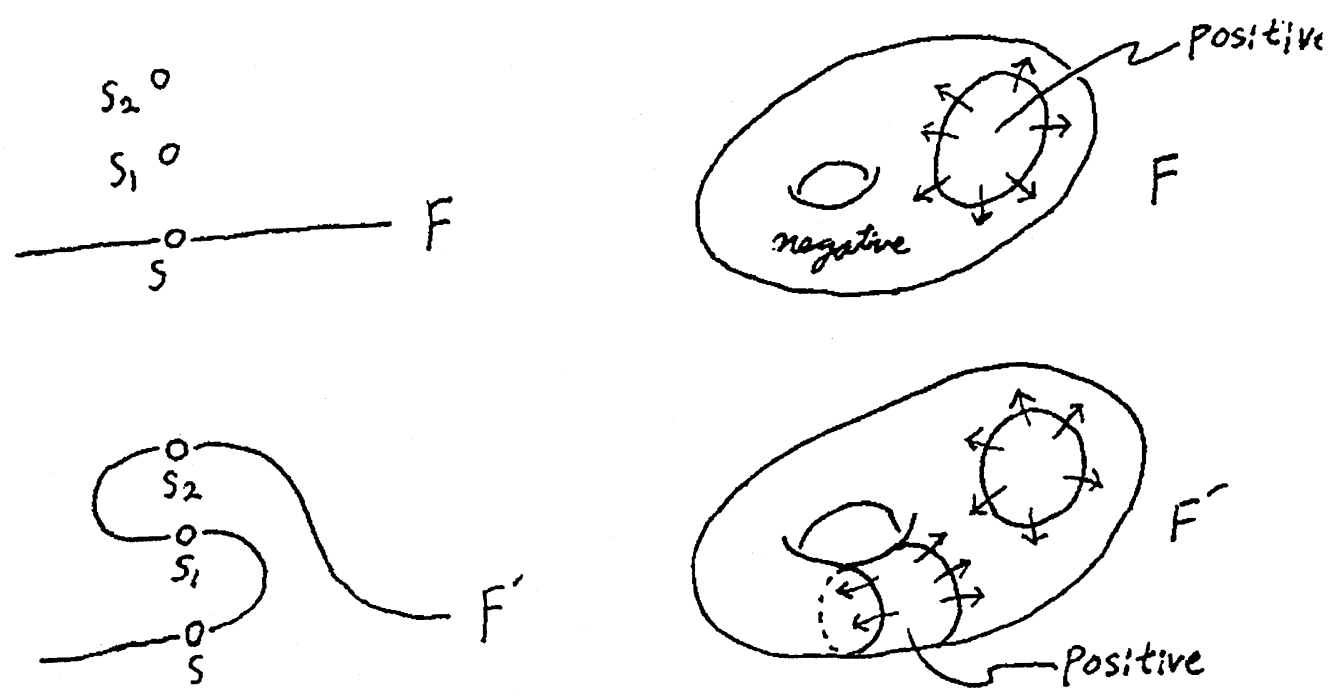

\section{Embedded Tori in Tight Contact 3-Manifolds.}

If the ambient contact 3-manifold is tight, the possible forms of the characteristic foliations on convex surfaces are quite limited.

Proposition 4.1 [G3]. Let $F$ be a closed connected convex surface with genus $(F)>0$ in a tight contact 3-manifold $(M, \zeta)$. Then no component of $F-\Gamma$ is homeomorphic to a disc.

Corollary. If genus $(F)=1$, the dividing set consists of even number of, parallel, non zero homotopic, simple closed curves.

Definition 4.2. Let $F$ be a convex torus in a tight contact 3-manifold $(M, \zeta)$.

$F$ is standard if the characteristic foliation $\operatorname{ch}_{\zeta}(F)$ is such that the pair $\left(F, c h_{\zeta}(F)\right)$ is diffeomorphic to $\left(\mathbb{R}^{2} / \mathbb{Z}^{2},[\sin 2 n \pi x \partial x]\right)$ for some non zero integer $n$, where $(x, y)$ are the natural coordinates of $\mathbb{R}^{2}$. We say that the simple closed curves corresponding to $\{0\} \times S^{1},\left\{\frac{1}{2 n}\right\} \times S^{1}, \ldots\left\{\frac{2 n-1}{2 n}\right\} \times S^{1}$ are horizontal Legendrian curves on the standard torus $F$. 
Figure 4.2.

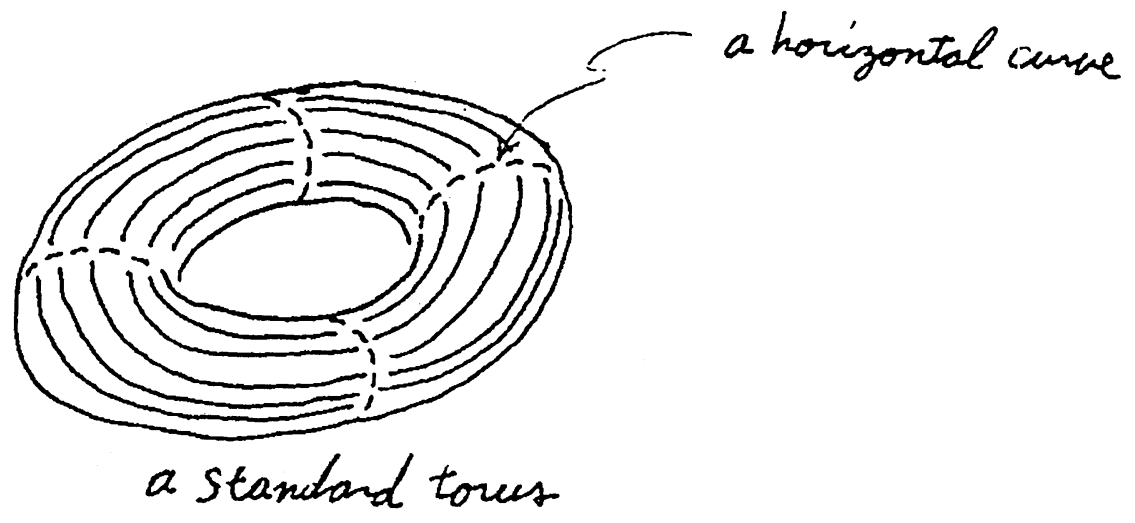

Proposition 4.3. Let $F$ be a convex torus in a tight contact 3-manifold $(M, \zeta)$. We can perturb $F$ by an admissible isotopy so that it is standard.

Proof. It is an easy consequence of Theorem 3.12 and Proposition 4.1.

\section{Legendrian Curves Contained in Convex Surfaces.}

Definition 5.1. An embedded curve $s$ in a contact 3-manifold. $(M, \zeta)$ is Legendrian if $s$ is tangent to the plane field $\zeta$.

Remark 5.2. A leaf of a characteristic foliation is a Legendrian curve.

Let $B(s)$ denote the normal bundle of $s$ in $M$. Then $\left.\zeta\right|_{s}$ gives a natural trivialization of $B(s)$.

Proposition 5.3 [G1], [W]. The isotopy classes of germs of pcsitive contact structure along $s$ are in one to one correspondence with the homotopy classes of trivializations of $B(s)$.

Lemma 5.4. Let $s$ be a simple closed Legendrian curve in $(M, \zeta)$ Define $V(s)$ to be the following subset of the set of sections of $B(s)$ :

$V(s):=\left\{\pi\left(\left.X\right|_{s}\right) \mid X\right.$ is a contact flow defined near $s$ such that $\left.X \pitchfork s\right\}$ 
where $\pi:\left.T M\right|_{s} \rightarrow B(s)$ is the natural projection. Define $G(s)$ to be the following subset of the set of smooth functions on s:

$$
G(s):=\left\{f \in C^{\infty}(s) \mid \text { if } f_{x}=0 \text { then } d f_{x} \neq 0\right\} .
$$

Then the defining 1-form $\alpha$ of $\zeta$ gives rise to an one to one map $\Psi_{\alpha}: V(s) \rightarrow$ $G(s)$ by $\Psi_{\alpha}(v)=\left.i(\bar{v}) \alpha\right|_{s}$ where $\pi(\bar{v})=v$.

Sketch of Proof. Let $f \in G(s)$ be given. Take an arbitrary extension $\stackrel{\sim}{\rightarrow} f$ of $f$ near $s$. The contact flow $\stackrel{\sim}{\rightarrow} v$ which satisfies $i(\stackrel{\sim}{\rightarrow} v) \alpha=\stackrel{\sim}{\rightarrow} f$ is given by the following equalities:

$$
\left\{\begin{array}{l}
i(\stackrel{\sim}{\rightarrow} v) \alpha=\stackrel{\sim}{\rightarrow} f \\
\left.i(\stackrel{\sim}{\rightarrow} v) d \alpha\right|_{\zeta}=-\left.d \stackrel{\sim}{\rightarrow} f\right|_{\zeta},
\end{array}\right.
$$

which is solved pointwise and uniquely with respect to $\stackrel{\sim}{\rightarrow} v$ thanks to the non integrability of $\zeta$. Then we may put $\Psi_{\alpha}^{-1}(f):=\pi\left(\left.\stackrel{\sim}{\rightarrow} v\right|_{s}\right)$.

The following Proposition plays an important role in the Proof of Theorem 0.3 .

Proposition 5.6. Let $s$ be a simple closed Legendrian curve contained in a convex surface $F$, and suppose that $\zeta$ is positive. Denote by $\operatorname{deg}_{\zeta}(s, F)$ the degree of $\left.T F\right|_{s}$ to the trivialization given by $\left.\zeta\right|_{s}$. Then it follows that.

$$
\operatorname{deg}_{\zeta}(v)=\frac{1}{2} \#\left(s \cap \Gamma_{F}\right) .
$$

Proof. Let $\alpha$ be a 1-form which defines $\zeta$. Take a contact flow $X$ transverse to $F$. The Lemma above and its proof implies that $\operatorname{deg}_{\zeta}(s, F)=$ $\left.\# i(X) \alpha\right|_{s} ^{-1}(0)$. On the other hand, $\left.i(X) \alpha\right|_{s} ^{-1}(0)=C(X) \cap F$ by the very definition of $C(X)$. Thus, the assartion is proven since $F \cap C(X)$ is a dividing set.

The following three Lemmas are the technical ones needed in Section 8.

Lemma 5.7. Let $s_{1}$ and $s_{2}$ be simple closed Legendrian curves contained in a closed orientable surface $F$ embedded in $(M, \zeta)$ such that $s_{1} \pitchfork s_{2} \subset F$. If a regular neighborhood $U \subset F$ of $s_{1} \cup s_{2}$ is convex, then we can perturb $F$ by a $C^{\infty}$-small isotopy which fixes $s_{1} \cup s_{2}$ so that $F$ is convex. 
The Lemma above is a slightly extended version of Theorem 3.8 and is proven in a way similar to it.

Definition 5.8. Let $s$ be a simple closed Legendrian curve contained in an embedded orientable surface $F$.

We say $F$ is standard along $s$ or $s$ is a standard curve on $F$ i:t there is a tubular neighborhood $U \subset F$ of $s$ such that the triple $\left(U, s, \mathrm{ch}_{\zeta}(U)\right)$ is diffeomorphic to $\left(S^{1} \times(-1,1), S^{1} \times\{0\},\left[\cos 2 \pi n \theta \partial_{\theta}\right]\right)$ for some non zero integer, where $\theta$ is the natural coordinate of $S^{1}$.

Lemma 5.9. Let $s$ be a simple closed Legendrian curve contained in a convex surface $F$. Then there is an embedded annulus $A$ which satisfies the following properties:

(1) $s$ is contained in the interior of $A$ as a standard curve,

(2) $A \pitchfork F$,

(3) $A \cap F=s$.

Proof. Since $F$ is convex, it is sufficient to consider only the case where $(M, F)$ is $(F \times \mathbb{R}, F \times\{0\})$ and $\zeta$ vertically invariant. Then $\zeta$ is defined by a 1-form $\beta+u d t$ where $\beta \in \Omega^{1}(F), u \in \Omega^{0}(F)$. We may take $s \times[-1,+1]$ as $A$.

Lemma 5.10. Let $s, F$ and $A$ be as in Proposition 5.9. Then we can perturb $F$ by a $C^{0}$-small admissible isotopy with not destroying the properties (1),(2) and (3) so that $F$ is standard along $s$.

Proof. All germs of positive (resp. negative ) contact structure around $F \cup A$ which induce the same characteristic foliation on $F \cup A$ are isotopic if $\partial A$ consists of two Legendrian curves. Therefore, we may assume that there is a contact flow $X$ which is transverse to $F$ and tangent to $A$.

Consider the unit circle bundle $U(s)$ associated to the normal bundle of $s$. Then $\left.X\right|_{s},\left.\zeta\right|_{s}$ and $\left.T F\right|_{s}$ determine sections of $U(s)$ denoted by $S_{X}, S_{\zeta}$ and $S_{T F}$, respectively. We can easily check that:

(1) $S_{X} \cap S_{T F}=\emptyset$,

(2) all the intersection points of $S_{X}$ and $S_{\zeta}$ are transverse and have the same sign. 
Therefore, we can perturb $F$ on the axis $s$ so that:

(1) $S_{X} \cap S_{T F}=\emptyset$,

(2) all the intersection points of $S_{T F}$ and $S_{\zeta}$ are transverse and have the same sign.

Of course, this perturbation admits the contact flow $X$.

As a result, the singular points of $c h_{\zeta}(F)$ on $s$ are finite and the signs of them occur alternately. Thus, if we modify the perturbation adequately in $C^{1}$-small manner, $\operatorname{ch}_{\zeta}(F)$ is standard along $s$.

\section{Vertically Invariant Contact Structures on $F \times \mathbb{R}$ and Contact Flows.}

Our aim in this section is to extend the admissible isotopy technique in Theorem 3.12 so that it is applicable to more generarized situation. Consider that two closed convex surfaces $F_{1}$ and $F_{2}$ intersect each other transversely. If we apply Theorem 3.12 directly to $F_{1}$ to deforme $\operatorname{chr}\left(F_{1}\right)$ in the exterior of a neighborhood of $F_{1} \cap F_{2}$, it is possible that the intersection of $F_{1}$ and $F_{2}$ changes i.e. new intersection points of them may appear. But if there exists a contact flow $X$ which has the following properties, no problem happens.

$$
\begin{aligned}
& \operatorname{supp}(X) \cap F_{2}=\emptyset . \\
& X \pitchfork F_{1} \text { except near } F_{1} \cap F_{2} . \\
& \text { Any orbit of } X \text { intersects } F_{1} \text { only once. }
\end{aligned}
$$

Proposition 6.1 and 6.2 assert that, if the intersection consists of either a dividing set or the union of standard curves of $F_{1}$, such a flow $X$ exists.

From now on, We consider contact flows on $F \times \mathbb{R}$ equipped with a vertically invariant contact structure.

Let $\zeta$ be a vertically invariant orientable contact structure on $F \times \mathbb{R}$. Then it can be defined by a 1 -form $\beta+u d t$ where $\beta \in \Omega^{1}(F), u \in \Omega^{0}(F)$.

The non integrability of $\zeta$ is equivalent to the condition that the 2-form $u d \beta+\beta \wedge d u$ on $F$ is nowhere zero.

Denote by $Y$ the flow on $F$ defined by the equation $i(Y) \omega=\beta$, where $\omega$ is a volume element on $F$. We regard $Y$ both as a flow on $F$ and as a vertically invariant, horizontal flow on $F \times \mathbb{R}$. Notice that $c h_{\zeta}(F \times\{t\})$ is generated by $Y$ for all $t$. 
By an easy calculation, we obtain the identity $u d \beta+\beta \wedge d u=\left(u \operatorname{div}_{\omega} Y-\right.$ $Y u) \omega$. Denote by $w$ the function $u \operatorname{div}_{\omega} Y-Y u$. Since $w$ is nowhere zero, we may assume that $w$ is positive.

Lemma 6.1. For any function $\Phi$ on $F \times \mathbb{R}$, there is the unique contact flow $W_{\Phi}$ with respect to $\zeta$ which satisfies the following equation:

$$
i\left(W_{\Phi}\right)(\beta+u d t)=\Phi u .
$$

Further, $W_{\Phi}$ satisfies the following equation:

$$
i\left(W_{\Phi}\right) d t=-\frac{u}{w}(Y \Phi)+\Phi .
$$

Proof. $W_{\phi}$ is contact if and only if the following equation is satisfied:

$$
\left.L_{W_{\phi}}(\beta+u d t)\right|_{\zeta}=0 .
$$

From (a) and (1), we obtain:

$$
\left.i\left(W_{\Phi}\right) d(\beta+u d t)\right|_{\zeta}=-\left.d(\Phi u)\right|_{\zeta} .
$$

The simultaneous equations (a) and (1) are equivalent to (a) and (2) which can be solved pointwise and uniquely.

Write $W_{\Phi}$ in the form $W+b \partial_{t}$ where $W$ and $b$ are a horizontal flow and a function on $F \times \mathbb{R}$, respectively. From (a) we obtain:

$$
\Phi u=i(W) \beta+b u .
$$

Applying $\frac{u}{w} i(Y)$ to the both sides of (2) and using the equations $\beta=i(Y) \omega$ and $d \beta=\left(\operatorname{div}_{\omega} Y\right) \omega$, we obtain:

$$
i(W) \beta=\frac{u^{2}}{w}(Y \Phi) .
$$

Applying it to (3), we obtain:

$$
u\left\{b-\left(-\frac{u}{w}(Y \Phi)+\Phi\right)\right\}=0 .
$$

Note that if $u_{x}=0$ then $d u_{x} \neq 0$ because $w=u d i v_{\omega} Y-Y u$ is nowhere zero. Therefore we obtain:

$$
b=-\frac{u}{w}(Y \Phi)+\Phi .
$$


We regard $F_{0}:=F \times 0$ and $F \times \mathbb{R}$ as a modell of a convex surface in a contact manifold. Then we can easily observe that $C\left(\partial_{t}\right)=\{(x, t) \mid u(x)=$ $0\}, F_{0}^{+, \partial_{t}}=\{(x, 0) \mid u(x)>0\}$ and $F_{0}^{-, \partial_{t}}=\{(x, 0) \mid u(x)<0\}$.

Let $\Gamma$ denote $\{x \in F \mid u(x)=0\}$.

Proposition 6.2. For any non-negative function $f$ on $F$ such that $f^{-1}(0)$ coincides with $\Gamma$, there is a contact flow $W$ such that:

(1) $W$ vanishes on a small tubular neighborhood $N$ of $\Gamma \times\{0\}$.

(2) $i(W) d t>0$ on $F_{0}-F_{0} \cap N$,

(3) $\operatorname{supp}(W) \subset S(f)$, where $S(f):=\{(x, t) \in F \times \mathbb{R}|| t \mid \leq f(x)\}$.

Proof. We have only to find a function $\Phi \in \Omega^{0}(F \times \mathbb{R})$ such that:

(1) $\operatorname{supp}(\Phi) \subset S(f)$,

(2) $-\frac{u}{w}(Y \Phi)+\Phi>0$ on $F_{0}-F_{0} \cap N$,

(3) $\Phi=0$ on $N$.

Then Lemma 5.1 implies that the contact flow $W:=W_{\Phi}$ (See Lemma 6.1) satisfies the required properties.

Denote by $\left\{\Gamma_{i}\right\}$ the connected components of $\Gamma$. Take a small tubular neighborhood $U_{i}$ of $\Gamma_{i} \subset F$ for each $i$ so that the triple $\left(U_{i}, \Gamma_{i},\left.Y\right|_{U_{i}}\right)$ is diffeomorphic to $\left((-1,1) \times S^{1},\{0\} \times S^{1}, \partial_{y}\right)$ where $y \in(-1,1)$.

Construct a function $\bar{\Phi}$ on $F$ so that it satisfies the following properties:

(1) $\bar{\Phi}=1$ on $F-U$, where $U:=\bigcup_{i} U_{i}$.

(2) $\bar{\Phi}>0$ on $F-U^{\prime}$, where $U^{\prime} \subset U$ is defined by $U^{\prime} \cap U_{i}=\left[-\frac{1}{2}, \frac{1}{2}\right] \times S^{1}$,

(3) $\bar{\Phi}=0$ on $U^{\prime}$,

(4) $\partial_{y}\left(\left.\bar{\Phi}\right|_{U_{i}}\right)\left\{\begin{array}{l}\leq 0 \text { on }\left(-1,-\frac{1}{2}\right) \times S^{1} \\ \geq 0 \text { on }\left(\frac{1}{2}, 1\right) \times S^{1}\end{array}\right.$ 
Then we see:

$$
-\frac{u}{w}(Y \bar{\Phi})+\bar{\Phi}\left\{\begin{array}{l}
>0 \text { on } F-U^{\prime} \\
=0 \text { on } U^{\prime}
\end{array}\right.
$$

Then we may define $\Phi$ by

$$
\Phi(x, t):=\bar{\Phi}(x) \cdot h(t),
$$

where $h$ is a function such that

$$
h(t)\left\{\begin{array}{l}
=0 \text { if }|t| \geq \sup _{x \in F-U^{\prime}} f \\
>0 \text { if otherwise }
\end{array}\right.
$$

Proposition 6.3. Let $s$ be a simple closed Legendrian curve contained in $F_{0}$. Suppose that $F_{0}$ is standard along $s$.

For any non-negative function $f$ on $F$ whose zero valued set coincides with $\pi(s)$ where $\pi F \times \mathbb{R} \rightarrow F$ is the natural projection, there exist a contact flow $W$ such that

(1) $i(W) d t$ is positive on $F_{0}-F_{0} \cap N$ where $N \subset F \times \mathbb{R}$ is a sufficiently small tubular neighborhood of $s$,

(2) $W=0$ on $N$,

(3) $\operatorname{supp}(W) \subset S(f)$.

Proof. Take a tubular neighborhood $U$ of $\pi(s) \subset F$ so that the triple $\left(U, \pi(s),\left.Y\right|_{U}\right)$ is diffeomorphic to $\left((-1,1) \times S^{1},\{0\} \times S^{1},\left[\cos 2 n \pi \theta \partial_{\theta}\right]\right)$, where $(x, \theta)$ are the coordinates of $(-1,1) \times S^{1}$.

Construct a function $\bar{\Phi}$ on $F$ so that it satisfies the following properties:

(1) $\bar{\Phi}=1$ on $F-U$,

(2) $\left.\bar{\Phi}\right|_{U}$ depends only on $x$,

(3) $\left.\bar{\Phi}\right|_{U}=0$ on $U^{\prime}$ where $U^{\prime}:=\left[-\frac{1}{2}, \frac{1}{2}\right] \times S^{1}$,

(4) $\bar{\Phi}>0$ on $F-U^{\prime}$. 
We may define the function $\Phi$ on $F \times \mathbb{R}$ in the same way as in the proof of Proposition 6.1. Since $\partial_{y}\left(\left.\bar{\Phi}\right|_{U}\right)=0, Y \Phi$ is identically zero on $F_{0}$. Therefore the function $-\frac{u}{a}(Y \Phi)+\Phi$ is positive on $F_{0}-U^{\prime} \times\{0\}$ and vanishes on $U^{\prime} \times \mathbb{R}$.

\section{The Invariant of $\left(T^{3}, \xi_{n}\right)$.}

We identify $T^{3}$ with $\mathbb{R}^{3} / \mathbb{Z}^{3}$. For each positive integer $n$, the contact structure $\xi_{n}$ is defined by the 1-form $\alpha_{n}:=\cos 2 n \pi z d x+\sin 2 n \pi z d y$. Give the orientation of $T^{3}$ by the 3 -form $d x \wedge d y \wedge d z$. Then $\xi_{n}$ is positive.

Definition 7.1. A simple closed curve $s$ in $T^{3}$ is linear if $s$ is isotopic to a simple closed curve given as the quotient of a "rational" line in $\mathbb{R}^{3}$ i.e. the one expressed by $(a t, b t, c t)$ where $t$ is the parameter and $(a, b, c) \in \mathbb{Q}^{3}-(0,0,0)$.

Definition 7,2. Let $s$ be a linear curve in $T^{3}$, and $B(s) \subset T^{3}$ the normal bundle of $s$. The canonical trivialization of $B(s)$ is the one given by $\left.T F\right|_{s}$ where $F$ is an incompressible torus which contains $s$. This definition is independent of the choice of $F$.

Definition 7.3. Let $s$ be a Legendrian linear curve in $\left(T^{3}, \zeta\right)$. The twisting number $t w(s)$ is minus the degree of $\left.\zeta\right|_{s}$ with respect to the canonical trivialization.

Lemma 7.4. Let $s$ be a Legendrian linear curve. For any positive integer $m$ there is a Legendrian linear curve $s^{\prime} C^{0}$-close to $s$ such that $t w\left(s^{\prime}\right)=$ $t w(s)+m$.

Proposition 7.5. Let $A$ be an element of $S L(3, \mathbb{Z})$. Let $\Psi_{A}$ denote the automorphism of $T^{3}$ induced by $A$.

If $\Psi_{A}$ preserves the 1-form $d z$, then $\Psi_{A}$ is isotopic to a contact automorphism of $\left(T^{3}, \xi_{n}\right)$ for any positive integer $n$.

Proof. By the assumption, $A$ is written as follows:

$$
A=\left(\begin{array}{lll}
a & c & e \\
b & d & f \\
0 & 0 & \delta
\end{array}\right),
$$

where $a, b, \ldots \delta \in \mathbb{Z}$ and $\operatorname{det} A=(a d-b c) \delta=1$. 
Denote $\Psi_{A}^{*}\left(\alpha_{n}\right)$ by $\alpha_{n}^{\prime}$. We have only to show that $\xi_{n}$ and the contact structure $\xi_{n}^{\prime}$ defined by $\alpha_{n}^{\prime}$ are connected by a smooth 1-parameter family of contact structures. Then the isotopy between them is easily constructed by Moser's method. See [W].

Put $H_{n, t}$ to be a 1-form with the parameter $t$ defined as follows:

$$
\begin{aligned}
H_{n, t}: & =(a(t) \cos 2 n \pi \delta z+b(t) \sin 2 n \pi \delta z) d x \\
& +(c(t) \cos 2 n \pi \delta z+d(t) \sin 2 n \pi \delta z) d y \\
& +(e(t) \cos 2 n \pi \delta z+f(t) \sin 2 n \pi \delta z) d z
\end{aligned}
$$

where $a(t), b(t), \ldots f(t)$ satiesfy

$$
\begin{aligned}
& a(0)=a, b(0)=b, \ldots f(0)=f \\
& a(1)=1, d(1)=\delta, b(1)=c(1)=e(1)=f(1)=0 .
\end{aligned}
$$

Then it follows that $H_{n, 0}=\alpha_{n}^{\prime}, H_{n, 1}=\delta \alpha_{n}$ and

$$
H_{n, t} \wedge d H_{n, t}=\delta(a(t) d(t)-b(t) c(t)) d x \wedge d y \wedge d z .
$$

Since $S L(2, \mathbb{R})$ is connected, we can take $a(t), b(t), c(t), d(t)$ so that they satisfy $\delta(a(t) d(t)-b(t) c(t))=1$ for all $t \in[0,1]$. Then $H_{n, t}$ gives a, contact homotopy between $\xi_{n}$ and $\xi_{n}^{\prime}$.

Theorem 7.6. Let $s$ be a Legendrian linear curve in $\left(T^{3}, \xi_{n}\right)$ such that $K[d z],[s]\rangle \mid>0$. Then the following inequality holds:

$$
t w(s) \geq|\langle[d z],[s]\rangle| \cdot n,
$$

where $\langle$,$\rangle is the Kronecker's product. Moreover this estimate is best possi-$ ble.

Proof. Case 1. $K[d z],[s]\rangle=1$

Proposition 7.5 implies that it is sufficient to consider only the case where $s$ is homotopic to the curve $s_{0}:=\{0\} \times\{0\} \times S^{1} \in T^{3}$. Let $\Psi_{a}: T^{3} \rightarrow T^{3}$ be the $a^{2}$-fold covering map defined by $\Psi_{a}(x, y, z)=(a x, a y, z)$. Notice that $\Psi_{a}^{-1}\left(\xi_{n}\right)=\xi_{n}$ Let $\stackrel{\sim}{\rightarrow} s$ be one of the lifts of $s$ with respect to $\Psi_{a}$. Then, since $\stackrel{\sim}{\rightarrow} s$ is a 1 -fold cover of $s$, it follows that $t w(\stackrel{\sim}{\rightarrow} s)=t w(s)$. Therefore we may assume that $s$ is contained in the subset $N_{\epsilon}$ defined by $N_{\epsilon}:=\left\{(x, y, z) \mid \sqrt{x^{2}+y^{2}}<\epsilon\right\} / \mathbb{Z}^{3}$, where $\epsilon<\frac{1}{2}$.

Now let $\left(S^{3}=S U(2), \xi_{0}\right)$ be the standard contact sphere described in Ex 2.3. 
Let $k$ be a closed orbit of the left invariant vector field $X$ in $S U(2)$. Then $k$ is a topologically trivial Legendrian knot in $S^{3}$. There is a tubular neighborhood $N^{\dagger}$ of $k$ and a contactmorphism $\Theta: N_{\varepsilon} \rightarrow N^{\dagger}$ such that $\Theta\left(s_{0}\right)=k$.

Let us get a contradiction by assuming $t w(s)<n$. By Lemma 7.1, we may suppose that $t w(s)=n-1$. Let $s^{\dagger}$ denote $\Theta(s)$. $s^{\dagger}$ is topologically trivial knot in $S^{3}$ and its Thurston-Bennequin invariant is zero i.e. if we move $s^{\dagger}$ slightly along the left invariant vector field $Z$ to a curve $s^{\ddagger}$, the linking number of $s^{\dagger}$ and $s^{\ddagger}$ is zero. Therefore there exists a Seifert disc $D$ of $s^{\dagger}$ such that $s^{\dagger}$ is a limit cycle of $c h_{\xi_{0}}(D)$. But this contradicts the tightness of $\left(S^{3}, \xi_{0}\right)$.

Case 2. $|\langle[d z],[s]\rangle|>1$

Put $b:=K[d z],[s]\rangle \mid$. Let $\Phi_{b}: T^{3} \rightarrow T^{3}$ be a $b$-fold covering map defined by $\Phi_{b}(x, y, z)=(x, y, b z)$. Notice that $\Phi_{b}^{-1}\left(\xi_{n}\right)=\xi_{b n}$.

Let $\stackrel{\sim}{\rightarrow} s$ be one of the lifts of $s$ with respect to $\Phi_{b}$. Then it follows that $t w(\stackrel{\sim}{\rightarrow} s)=t w(s)$. On the other hand, the result of Case 1 implies that $t w(\stackrel{\sim}{\rightarrow} s) \geq b n$.

Corollary 7.7. If $f:\left(T^{3}, \xi_{n}\right) \rightarrow\left(T^{3}, \xi_{m}\right)$ is a contact diffeomorphism, then $n=m$ and $f$ is isotopic to an automorphism which preserves the 1form $d z$.

\section{The Classification of Tight Contact Structures in $T^{3}$.}

Theorem 8.1. Let $\zeta$ be an orientable positive tight contact structure on the 3-torus $T^{3}$. Then there are a positive number $n$ and an element $f$ of $\operatorname{Diff}^{+}\left(T^{3}\right)$ such that $f:\left(T^{3}, \zeta\right) \rightarrow\left(T^{3}, \xi_{n}\right)$ is a contactmorphism.

The following two theorems are necessary for proving Theorem 8.1.

Theorem 8.2. Let $G$ be a solid torus. Let $\mathfrak{F}$ be a singular foliation on $\partial G$ which satisfies the following properties:

(1) There is a subset $\Gamma$ of $\partial G$ which divides $\mathfrak{F}$,

(2) $\# \Gamma=2$,

(3) Each component of $\Gamma$ is homotopically equivalent to $G$ in $G$ i.e. $\Gamma$ is the disjoint union of Longitudes. 
If two positive (resp. negative) tight contact structures on $G$ induce the same characteristic foliation $\mathfrak{F}$ on $\partial G$, then they are isotopic relative to $\partial G$.

Definition 8.3. Let $T$ be a convex torus in a tight contact 3-manifold. The torus division number of $T$ denoted by $t d(T)$ is the number $\frac{1}{2} \#\left(\Gamma_{T}\right)$.

By Proposition 4.1, $t d(T)$ must be an integer.

Theorem 8.4. Let $\zeta$ be an orientable tight contact structure on the 3-torus $T^{3}$. Then there are two incompressible convex tori $S$ and $T$ in $T^{3}$ such that the following properties are satisfied:

(1) $S \pitchfork T$ and the intersection of them consists of a Legendrian simple closed curve $s$,

(2) Both $S$ and $T$ are standard,

(3) $t d(S)=t d(T)=t w(s)$,

(4) Each component of $\Gamma_{S}$ and $\Gamma_{T}$ is not homotopic to $s$.

Proof of Theorem 8.1. Let $S$ and $T$ be as expressed in Theorem 8.4. There is a germ of contactmorphism $f$ which maps $S \cup T$ to $\left\{(0, y, z) \mid y, z \in S^{1}\right\} \cup$ $\left\{(x, 0, z) \mid x, z \in S^{1}\right\} \subset\left(T^{3}, \xi_{t w(s)}\right)$. We can take a sufficiently small regular neighborhood $N$ of $S \cup T$ so that $\partial \bar{N}$ is a convex torus and so that the solid torus $T^{3}-N$ satisfies the assumptions of Theorem 8.2.

For example, consider an embedded circle $C$ in the interior of $[0,1] \times[0,1]$ such that $C$ is contained in a small regular neighborhood of $\partial([0,1] \times[0,1])$ and its normal curvature is positive. Embed $(0,1) \times(0,1) \times S^{1}$ naturally in $\left(T^{3}, \xi_{t w(s)}\right)$. If we take $N$ so that $\partial N=C \times S^{1}, N$ is the required one. Notice that in this case, the singularities of $c h_{\xi_{t w(s)}}(\partial N)$ consistis of two disjoint Legendrian simple closed curves.

Therefore $f$ extends to a contactmorphism from the whole $T^{3}$ to $T^{3}$ by Theorem 8.2.

Proof of Theorem 8.2. Since $\partial G$ is convex, there is a contact flow $X$ on $G$ which is transverse to $\partial G$ and goes inward along $\partial G$. We can take an embedding $\Phi: T^{2} \times \mathbb{R}^{\geq 0} \rightarrow G$ so that:

(1) $\Phi\left(T^{2} \times\{0\}\right)=\partial G$, 
(2) $\Phi_{*}\left(\partial_{t}\right)=X$,

(3) $\Phi^{-1}(\zeta)$ is vertically invariant with respect to $\partial_{t}$.

If we take a positive function $h$ on $T^{2}$ approprietly, its graph $H:=$ $\{(x, h(x))\} \in T^{2} \times \mathbb{R}^{\geq 0}$ satisfies the following:

(1) $H$ is a standard torus,

(2) $\Phi$ (the standard Legendrian curves of $H$ ) are null homotopic in $G$.

Let $l$ be one of the standard Legendrian curves of $H$. Denote by $G^{\prime}$ the set $G-\Phi(\{(x, t) \mid 0 \leq t<h(x)\})$. Proposition 5.9 implies that we can take a convex disc $D \subset G^{\prime}$ so that:

(1) $\Phi(l)=D \cap \partial G^{\prime}=\partial D$,

(2) $D \pitchfork \partial G^{\prime}$

(3) $D$ is standard along $\partial D$.

Since $\zeta$ is tight, $\Gamma_{D}$ consists of an arc with its ends on $\partial D$. We can perturb $D$ by an admissible isotopy which is the identity near $\partial D$ so that $c h_{\zeta}(D)$ is in the form shown in Fig.8.1. Since Int $G^{\prime}-D$ is homeomorphic to the open 3-ball, and the germ of contact structure along $\partial G^{\prime} \cup D$ is unique up to isotopy, $\left.\zeta\right|_{G^{\prime}}$ is also unique up to isotopy by Theorem 2.5 .

Obviously, we can choose $\zeta^{\prime}, h$ and $\operatorname{ch}_{\zeta}(D)$ to be an fixed model with respect to the given $\mathfrak{F}$. Therefore the tight contact structure on $G$ is unique up to isotopy to the given $\mathfrak{F}$.

Proof of Theorem 8.4. Let $E$ be an incompressible torus in $\left(T^{3}, \zeta\right)$ and have the least torus division number of all such tori. We may assume by Proposition 4.3 that $E$ is standard.

Let $s_{1}$ be a linear Legendrian curve in $\left(T^{3}, \zeta\right)$ which intersect $E$ only once and transversely such that it has the least non negative twist number of all such curves.

Take an incompressible torus $F$ so that

(1) $F$ contains $s_{1}$,

(2) $F$ intersects $E$ transversely,

(3) $E \cap F$ consists of a Legendrian curve $s_{0}$ which is one of the standard Legendrian curves on $E$. 
Figure 8.1.

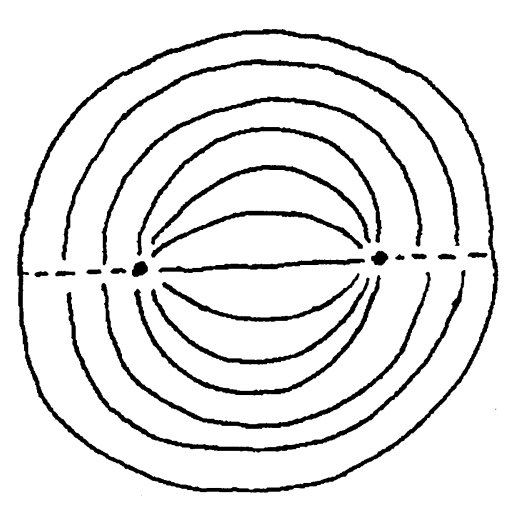

Then we can make $F$ convex in the following way:

Since $E$ is convex, there is a contact flow $X$ defined only near $E$, so that $X \pitchfork E$. By choosing $X$ generic, we may assume that $X$ is not tangent to $s_{1}$. We can perturb $F$ on the axis $s_{0}$ so that $F \pitchfork X$. Since $t w\left(s_{1}\right) \geq 0$, we can extend $X$ to along $s_{1}$ so that $X \pitchfork s_{1}$ and so that $\left.X\right|_{s_{1}}$ may give the canonical trivialization to the normal bundle of $s_{1}$. We can perturb $F$ on the axis $s_{1}$ so that $\left.F \pitchfork X\right|_{s_{1}}$. Proposition 5.7 implies that we can rnake the whole $F$ convex through $C^{\infty}$-small isotopy.

Case 1) Each component of $\Gamma_{F}$ is not homotopic to $s_{0}$.

We will show that $t d(E)=t d(F)$. Since each component of $\Gamma_{F}$ intersects $s_{0}$ at least once by the assumption, we see that $t w\left(s_{0}\right)=\frac{1}{2} \#\left(\Gamma_{F} \cap s_{0}\right) \geq$ $\frac{1}{2} \#\left(\Gamma_{F}\right)=t d(F)$. (See Proposition 5.6.) On the other hand, $t w\left(s_{0}\right)=$ $\frac{1}{2} \#\left(\Gamma_{E} \cap s_{0}\right)=\frac{1}{2} \# \Gamma_{E}=t d(E)$. Remember that $t d(E)$ is minimal. Therefore, $t d(E)=t d(F)$.

Since $E$ is standard, Proposition 5.10 implies that $F$ can be perturbed so that it is standard at least near $s_{0}$. Then Proposition 6.3 and Theorem 3.2 implies that the whole $F$ can be perturbed so that it is standard. Therefore, $E$ and $F$ satisfy the required properties.

Case 2) Each component of $\Gamma_{F}$ is homotopic to $s_{0}$.

Step 1) We will show that $t d(E)=1$.

Let us get a contradiction by assuming $t d(E)>1$.

Make $F$ standard along $s_{0}$ by Proposition 5.10. There is a component $A$ of $F-s_{0} \cup \Gamma_{F}$ such that $A$ is homeomorphic to a disc and such that $\#\left(\partial \bar{A} \cap s_{0}\right)=\#\left(\partial \bar{A} \cap \Gamma_{F}\right)=1$. We may suppose that $A$ is contained in $F^{+}$. 


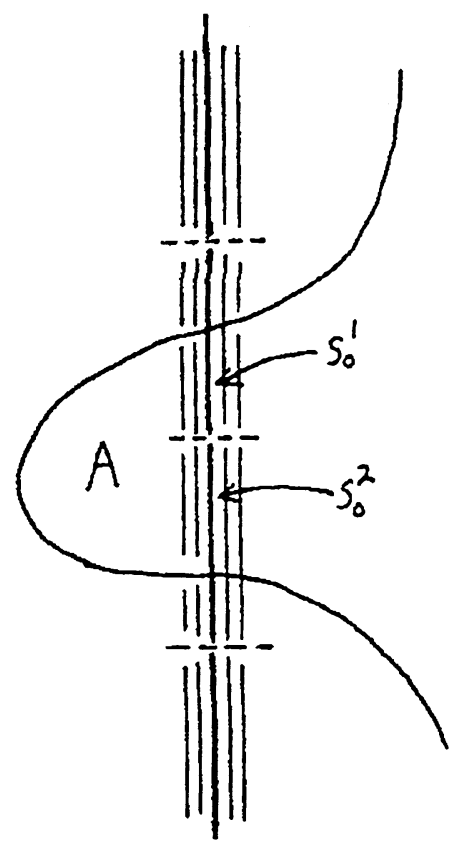

Figure 8.2.

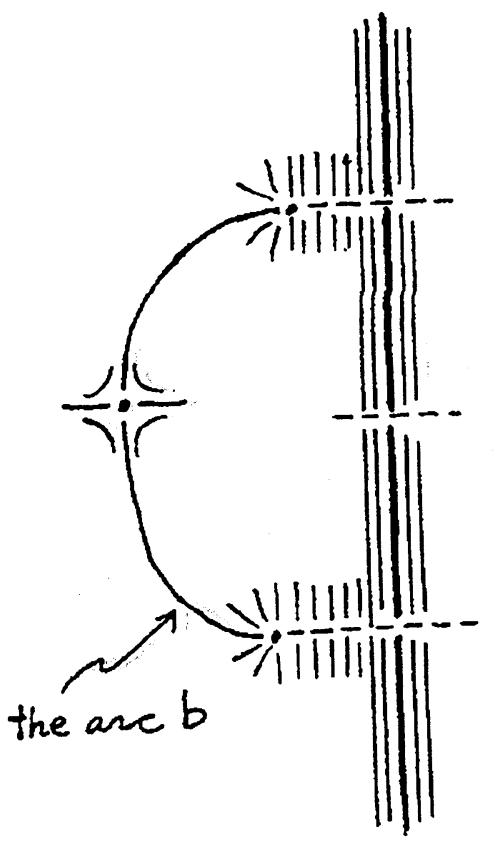

There is a contact flow $X$ such that:

(1) $X \pitchfork(F-\bar{V})$,

(2) $X$ vanishes on $\bar{V}$,

(3) $\operatorname{supp}(X) \cap E=\emptyset$,

where $V \subset F$ denotes a sufficiently small tubular neighborhood of $s_{0}$. (See Proposition 6.3.) Denote by $p_{1}$ and $p_{2}$ the two singular points of $\operatorname{ch}_{\zeta}\left(F^{\prime}\right)$ on $s_{0}$ that neighbors the only one singular point on $s_{0} \cap \bar{A}$. Deform $F$ to $F^{\prime}$ by an admissible isotopy with respect to $X$ so that $\left(F^{\prime}\right)^{-}$contains a Legendrian arc $b$ which connects $p_{1}$ and $p_{2}$. See Fig 8.2. Since the number of leaves of $\operatorname{ch}_{\zeta}\left(F^{\prime}\right)$ on $s_{0}$ is equal to $2 t w\left(s_{0}\right)=2 t d(E) \geq 4$ and only two of them, denoted by $s_{0}^{1}$ and $s_{0}^{2}$, intersect $\bar{A}$, we can chose a leaf $s_{0}^{0}$ on $s_{0}$ such that $s_{0}^{0} \cap \bar{A}=\emptyset$. Denote by $k$ the horizontal Legendrian curve on $E$ which intersect $s_{0}^{0}$.

Denote by $s_{0}^{\prime}$ the closed Legendrian curve with corners $\overline{b \cup\left(s_{0}-\overline{s_{0}^{1} \cup s_{0}^{2}}\right)}$. We can deform it in a $C^{0}$-small manner to a curve with no corner so that $t w\left(s_{0}^{\prime}\right)=t w\left(s_{0}\right)-1$.

Take an incompressible torus $E^{\prime}$ which contains $s_{0}^{\prime} \cup k$. We can make $E^{\prime}$ convex in a neighborhood of $s_{0}^{\prime} \cup k$ in the following way. 
Since $t w\left(s_{0}\right)>0$ and $t w(k)=0$, we can take a contact flow $X$ along $s_{0}^{\prime} \cup k$ so that:

(1) $X \pitchfork\left(s_{0}^{\prime} \cup k\right)$,

(2) $\left.X\right|_{s_{0}^{\prime}}$ and $\left.X\right|_{k}$ give the canonical trivializations to the normal bundles of $s_{0}^{\gamma}$ and $k$, respectively.

Perturb $E^{\prime}$ so that $E^{\prime} \pitchfork X$ near $s_{0}^{\prime} \cup k$. Then, Proposition 5.7 implies that we can deform the whole $E^{\prime}$ to a convex torus.

Since $t w(k)=0, k$ does not intersect $\Gamma_{E^{\prime}}$, Therefore, $t d\left(E^{\prime}\right)=$ $\frac{1}{2} \#\left(\Gamma_{E^{\prime}}\right) \leq \frac{1}{2} \#\left(\Gamma_{E^{\prime}} \cap s_{0}^{\prime}\right)=t w\left(s_{0}^{\prime}\right)=t d(E)-1$. But this contradicts the minimality of $t d(E)$.

Step 2) We will show that $t d(F)=t w\left(s_{1}\right)$.

Since each component of $\Gamma_{F}$ is homotopic to $s_{0}$, we obtain $\operatorname{td}(F) \leq$ $t w\left(s_{1}\right)$.

Let us get a contradiction by assuming $t d(F)<t w\left(s_{1}\right)$. From Step 1) it follows that only one component $f_{0}$ of $\Gamma_{F}$ intersects $s_{0}$ and \# $\left(s_{0} \cap f_{0}\right)=2$. Therefore, there is a (not Legendrian in general) simple closed curve $s_{1}^{\prime}$ on $F$ such that

(1) $s_{1}^{\prime} \pitchfork \Gamma_{F}$ and \# $\left(s_{1}^{\prime} \cap\left(\right.\right.$ each component of $\left.\left.\Gamma_{F}\right)\right)=1$,

(2) $s_{1}^{\prime} \pitchfork s_{0}$ and $\#\left(s_{1}^{\prime} \cap s_{0}\right)=1$,

(3) If $V \subset F$ is a sufficiently small tubular neighborhood of $s_{0}, V \cap s_{1}^{\prime}$ is a Legendrian arc which connects the two component of $\partial \bar{V}$.

Proposition 6.3 implies that we can take a contact flow $X$ so that:

(1) $X \pitchfork F-\overline{V^{\prime}}$,

(2) $X=0$ on $\overline{V^{\prime}}$,

(3) $\operatorname{supp}(X) \cap E=\emptyset$,

where $V^{\prime} \subset V$ is a tubular neighborhood of $s_{0}$ such that $\overline{V^{\prime}}(-V$. By an admissible isotopy with respect to $X$, we can perturb $F$ so that $s_{1}^{\prime}$ is realized as a Legendrian simple closed curve contained in $F$. Then it follows that $t w\left(s_{1}^{\prime}\right)=\frac{1}{2} \#\left(s_{1}^{\prime} \cap \Gamma_{F}\right)=t d(F)<t w\left(s_{1}\right)$. But this contradicts the minimality of $t w\left(s_{1}\right)$.

Step 3) Take an incompressible torus $G$ such that

(1) $G \pitchfork E$, 
(2) $G \cap E$ consists of a simple closed curve $s_{2}$ such that $s_{2} \pitchfork \zeta$,

(3) $G \pitchfork F$ and $G \cap F=s_{1}$,

(4) $G$ is standard along $s_{1}$.

Proposition 5.9 guarantees that we can take $G$ so that it satisfies the property (4).

We assert that we can perturb $G$ so that it satisfies the following:

(1) $G$ is convex,

(2) One of the components of $\Gamma_{G}$ coincides with $s_{2}$,

(3) $t d(G)=t w\left(s_{1}\right)$.

To prove the assertion above, we will divide our argument into the following four small steps.

Step 1) Take a sufficiently small tubular neighborhood $N$ of $E$. We can identify the 5-tuple

$$
\left(N, E, N \cap F, N \cap G,\left.\zeta\right|_{N}\right)
$$

with

$$
\begin{aligned}
&\left(S^{1} \times S^{1} \times(-\epsilon, \epsilon), S^{1} \times S^{1} \times\{0\}, S^{1} \times\{0\} \times(-\epsilon, \epsilon),\right. \\
&\{0\}\left.\times S^{1} \times(-\epsilon, \epsilon), \cos 2 \pi x d y+\sin 2 \pi x d z=0\right)
\end{aligned}
$$

where $(x, y, z)$ are the coordinates of $S^{1} \times S^{1} \times(-\epsilon, \epsilon)$.

Take an incompressible torus $G^{\prime}$ so that

(1) $G^{\prime} \cap\left(T^{3}-N\right)=G \cap\left(T^{3}-N\right)$.

(2) $G^{\prime} \cap N=\left\{(g(z), y, z) \mid y \in S^{1}, z \in(-\epsilon, \epsilon)\right\}$, where $g$ is a function such that

(a) $g \in C_{0}^{\infty}(-\epsilon, \epsilon)$,

(b) $g$ is monotonically increasing (resp. decreasing) on $\left(-\frac{1}{2} \epsilon, 0\right)$ (resp. on $\left.\left(0, \frac{1}{2} \epsilon\right)\right)$,

(c) $\operatorname{supp}(g)=\left[-\frac{1}{2} \epsilon, \frac{1}{2} \epsilon\right]$,

(d) $g(0)=\frac{1}{2}$.

See Fig.8.3.

Let $A$ denote the annulus $G^{\prime} \cap N$. Then $\operatorname{ch}_{\zeta}(A)$ satisfies the following: 
Figure 8.3.

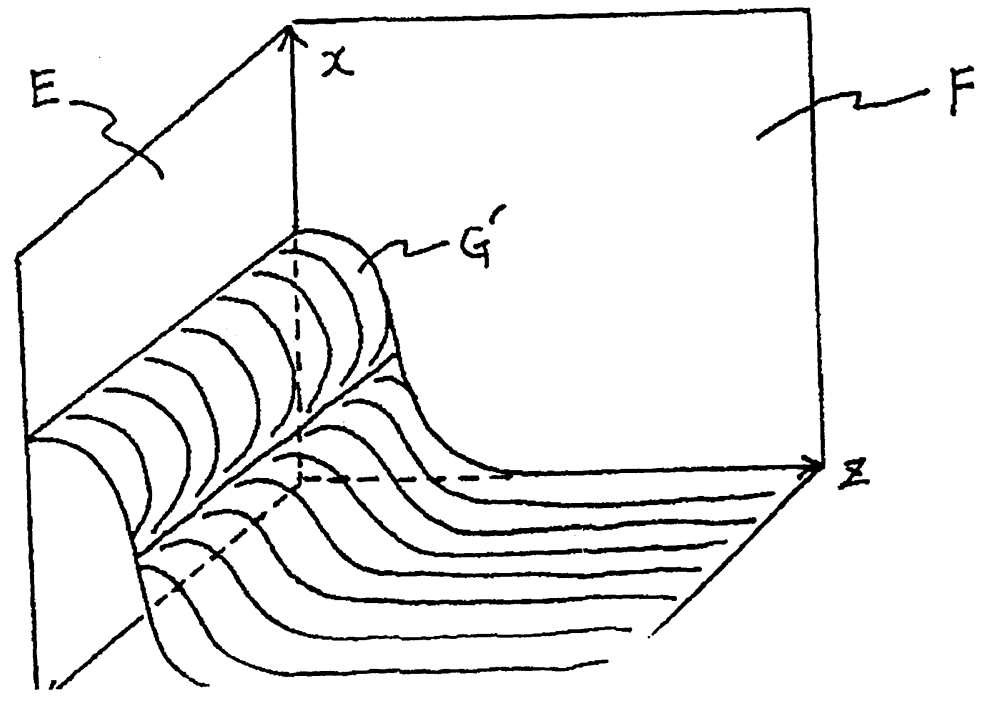

(1) $\operatorname{ch}_{\zeta}(A)$ is induced by a Morse-Smale flow $Y$,

(2) $Y$ is non singular,

(3) $Y \pitchfork \partial A$,

(4) $Y$ has only two closed orbit.

We can perturb $G^{\prime}$ through a $C^{\infty}$-small isotopy which is the identity both in $G^{\prime} \cap N$ and in a sufficiently small tubular neighborhood of $s_{1}$ so that it is convex. Then we can choose $s_{2}$ as one of the components of $\Gamma_{G^{\prime}}$ Step 2) Let us show that $t d\left(G^{\prime}\right) \geq t w\left(s_{1}\right)+1$.

Assume that $t d\left(G^{\prime}\right)<t w\left(s_{1}\right)+1$. Then we can perturb $G^{\prime}$ through an admmisible isotopy $H_{t}$ so that $G^{\prime}$ contain a Legendrian curve $l$ which satiesfies the following:

(1) $l \pitchfork \Gamma_{G^{\prime}}$, and $l$ intersects each component of $\Gamma_{G^{\prime}}$ except $s_{2}$ only once,

(2) $\bar{l}-l$ consists of the two closed orbit in $A$,

(3) $l \cap \partial \bar{N}$ consists of the two points expressed by $\left(0, y_{0}, \epsilon\right)$ and $\left(0, y_{0},-\epsilon\right)$ for some $y_{0} \in S^{1}$ with respect to the coordinates of $\bar{N}$, 
Further, the isotopy $H_{t}$ can be taken so that it is the identity in $G^{\prime} \cap N$, $\operatorname{Im}\left(H_{t}\right) \cap E=s_{2}$ and $H_{t}^{-1}\left(s_{2}\right)=s_{2}$, for all $t$. (See Proposition 6.2.)

Let $s_{1}$ denote the linear Legendrian curve $(l-l \cap N) \cup\left\{\left(0, y_{0}, z\right)|| z \mid<\epsilon\right\}$. Then $t w\left(\dot{s}_{1}\right)=t d\left(G^{\prime}\right)-1$ since $\zeta_{\dot{s}_{1}}$ hardly rotates on $\dot{s}_{1} \cap N$ and since the property (1) above holds. Therefore $t w\left(s_{1}\right)<t w\left(s_{1}\right)$. But it contradicts the minimality of $t w\left(s_{1}\right)$

Step 3) Let $A_{1}$ and $A_{2}$ denote the two components of $G^{\prime}-\Gamma_{G^{\prime}}$ such that both $\partial \bar{A}_{1}$ and $\partial \bar{A}_{2}$ contain $s_{2}$. Since $t d\left(G^{\prime}\right)=t w\left(s_{1}\right)+1=t d(F)+1 \geq 2$, we see that $\bar{A}_{1} \cap \bar{A}_{2}=s_{2}$. We will eliminate all the singularities of $\operatorname{ch}_{\zeta}\left(A_{1} \cup A_{2}\right)$.

We may assume that $\operatorname{ch}_{\zeta}\left(G^{\prime}\right)$ is generated by a Morse-Smale flow $Y$. Since each $A_{i}$ contains a closed orbit of its characteristic foliation in $A_{i} \cap A$, $A_{i}$ does not have another closed orbit. Further, there is no singular points in $A_{i} \cap A$. We can eliminate all the singular points of $\operatorname{ch}_{\zeta}\left(A_{i}\right)$ in the following way.

We may assume $A_{1}$ positive. Let $e$ be an elliptic point in $A_{1}$. Of course, the divergence of $Y$ at $e$ is positive. If any orbit which comes from $e$ does not reach any hyperbolic points, all such orbits must reach the boundary component of $\overline{A_{1}}$ other than $s_{2}$, which implies that $A_{1}$ is homeomorphic to a disc and gives rise to a contradiction. Therefore, at least one hyperbolic point denoted by $h$ is connected with $e$ by a separatrix $b$. Proposition 3.13 implies that we can eliminate $e$ and $h$ together by perturbing $A_{1}$ only near $e \cup b \cup h$ in a $C^{0}$-small way. Since the Euler number of $A_{1}$ is zero, $\operatorname{ch}_{\zeta}\left(A_{1}\right)$ can be made non-singular. $\operatorname{ch}_{\zeta}\left(A_{2}\right)$ can be made non-singular in the same way.

Step 4) Perturb $G$ so that $G \cap N^{c}$ coincides with $G^{\prime} \cap N^{c}$.

Let $B$ denote the annulus $\left(\left(A_{1} \cup A_{2}\right) \cap G\right) \cup(N \cap G)$. Then $c h_{\zeta}(G)$ is as follows:

(1) Every leaf of $\operatorname{ch}_{\zeta}(B)$ is an arc which connects the two components of $\partial \bar{B}$.

(2) $G-B$ is a convex annulus such that

1) $\partial(G-B) \pitchfork \operatorname{ch}_{\zeta}(G-B)$.

2) If $Y^{\prime}$ generates $c h_{\zeta}(G-B), Y^{\prime}$ goes inward along one component of $\partial(G-B)$ which bounds the negative part of $G-B$, and outward along the other which bounds the positive part of $G-B$.

Therefore, we can choose the dividing set $\Gamma_{G}$ so that $s_{2}$ is one of the components of $\Gamma_{G}$. Then $t d(G)=t d\left(G^{\prime}\right)-1=t w\left(s_{1}\right)$ since $s_{1}$ intersects each component of $\Gamma_{G}$ at least once. 
Step 5) Remember that $G$ is standard along $s_{1}$. Then we can make $F$ standard along $s_{1}$ by Proposition 5.10. Then the whole $G$ and the whole $F$ can be made standard by Proposition 6.3 so that they satisfy the required properties.

\section{References.}

[A] V.I. Arnold, Méthodes mathématiques de la mécanique classique, Mir 1974.

[E1] Ya. Eliashberg, Classification of over twisted contact structures on 3manifolds, InventMath'98 (1989), 623-637.

[E2] - Contact 3-manifolds, twenty years since J.Martinet's work, AnnInstFiourier, 42 (1992), 165-192.

[G1] E. Giroux, Convexité en topologie de contact, MathḢelv'66 (1991), 637-677.

[G2] Une structure de contact, même tendue, est plus ou moins tordue, prépublication (E.N.S.-Lyon 1992).

[W] A. Weinstein, Lectures on Symplectic Manifolds, C.B.M.S No.29.

RECEIVEd MAY 25, 1995.

UNIVERSITY HOKKAIDO

DEPARTMENT OF MATHEMATICS

FACULTY OF SCIENCES

Kita 10 Jyou Nishi 8 Choume, KitakU

SAPPOROSHI

JAPAN

E-mail address: KANDA@MATH.HOKKAIDO.AC.JP. 